Trinity University

Digital Commons@ Trinity

Mathematics Faculty Research

Mathematics Department

$4-2007$

\title{
Population Models in Almost Periodic Environments
}

Toka Diagana

Saber Elaydi

Trinity University, selaydi@trinity.edu

Abdul-Aziz Yakubu

Follow this and additional works at: https://digitalcommons.trinity.edu/math_faculty

Part of the Mathematics Commons

\section{Repository Citation}

Diagana, T., Elaydi, S., \& Yakubu, A-A. (2007). Population models in almost periodic environments. Journal of Difference Equations and Applications, 13(4), 239-260. doi:10.1080/10236190601079035

This Post-Print is brought to you for free and open access by the Mathematics Department at Digital Commons @ Trinity. It has been accepted for inclusion in Mathematics Faculty Research by an authorized administrator of Digital Commons @ Trinity. For more information, please contact jcostanz@trinity.edu. 


\title{
POPULATION MODELS IN ALMOST PERIODIC ENVIRONMENTS
}

\author{
TOKA DIAGANA, SABER ELAYDI, AND ABDUL-AZIZ YAKUBU
}

\begin{abstract}
We establish the basic theory of almost periodic sequences on $\mathbb{Z}_{+}$. Dichotomy techniques are then utilized to find sufficient conditions for the existence of a globally attracting almost periodic solution of a semilinear system of difference equations. These existence results are, subsequently, applied to discretely reproducing populations with and without overlapping generations. Furthermore, we access evidence for attenuance and resonance in almost periodically forced population models.
\end{abstract}

\section{INTRODUCTION}

Despite the fact that all natural populations suffer temporal environmental fluctuations on some scale, experimental and theoretical studies of population responses to external fluctuations remain relatively rare $[8,9,10]$, [12, 13, 14, 15, 16, 17], $[20,21,22,23,24],[28,29,30],[32]$, and $[35,36,36,38,39,40,41]$. To study the effects of fluctuating environments on population dynamics, one can deliberately fluctuate environmental parameters in controlled laboratory experiments. Jillson performed such an experiment with laboratory cultures of Tribolium [34]. Others have used mathematical models to study the effects of periodic forcing on population dynamics $[8,9,10],[12,13,14,15,16,17],[20,21,22,23,24],[29,30]$, and $[35,36,37,38]$.

Though one can deliberately periodically fluctuate environmental parameters in controlled laboratory experiments, fluctuations in nature are hardly periodic. That is, almost periodicity is more likely to accurately describe natural fluctuations. Almost periodicity has been observed in data collected by Henson et al. on tidal heights [31]. The ecological literature is filled with future life-history studies of periodically forced (nonautonomous) classical parametric population models with time $t$ in $\mathbb{Z}_{+}$. Examples of such periodically forced parametric models with time $\mathrm{t}$ in $\mathbb{Z}_{+}$include the Beverton-Holt, Ricker, Smith-Slatkin and LPA models [9, 10], $[13,14,15,16]$, and $[21,22,23,24]$.

In this paper, we focus on the effects of almost periodic environments on population dynamics. That is, we study future life-history evolutions in these environments with time $t \in \mathbb{Z}+$. For that, we make extensive use of dichotomy techniques to find sufficient conditions for the existence of a globally attracting almost periodic solution to a semilinear system of difference equations. These existence results are applied to discretely reproducing populations with and without overlapping generations.

2000 Mathematics Subject Classification. 34C27; 34K14; 34D23.

Key words and phrases. Bohr almost periodic sequences, Bochner almost periodic sequences, almost periodicity, regular dichotomy, globally attracting almost periodic solution, Beverton-Holt equation, Beverton-Holt equation with delay. 
The paper is organized as follows: in Section 2, we establish the theory of almost periodic sequences on $\mathbb{Z}_{+}$. In particular, we show that the notions of Bohr and Bochner almost periodic sequences on $\mathbb{Z}_{+}$are equivalent. In Section 3 , we provide a method to construct almost periodic sequences on $\mathbb{Z}_{+}$. Sufficient conditions for the existence of a globally attracting almost periodic solution of a semilinear system of difference equations are given in Section 4 (Theorem 4.6). In Section 5 (respectively, Section 6), we apply Theorem 4.6 to population models with nonoverlapping generations (respectively, overlapping generations). Section 7 is on accessing evidence of attenuance and resonance in almost periodic environments. Extensions to population models with delay are developed in Sections 8 and 9, and the implications of our results are discussed in Section 10.

\section{Preliminaries}

In this section, we establish the basic theory of almost periodic sequences on $\mathbb{Z}_{+}$. But first, let us introduce the notation needed in the sequel.

Let $(\mathbb{R},|\cdot|),\left(\mathbb{R}^{k},|\cdot|\right), \mathbb{R}_{+}, \mathbb{Z}, \mathbb{Z}_{+}$be the field of real numbers equipped with its absolute value, the $k$-dimensional space of real numbers equipped with the Euclidean topology, the set of positive real numbers, the set of all integers, and the set of all nonnegative integers, respectively.

Our main objective in this paper is to find sufficient conditions for the existence of a globally attracting almost periodic solution of the semilinear systems of difference equations

$$
x(t+1)=A(t) x(t)+f(t, x(t)), \quad t \in \mathbb{Z}_{+}
$$

where $A(t)$ is a $k \times k$ almost periodic matrix function defined on $\mathbb{Z}_{+}$, and the function $f: \mathbb{Z}_{+} \times \mathbb{R}^{k} \rightarrow \mathbb{R}^{k}$ is almost periodic.

To study solutions of Eq. (2.1), we use the fundamental solutions of the system

$$
x(t+1)=A(t) x(t), \quad t \in \mathbb{Z}_{+},
$$

to examine almost periodic solutions of the system of difference equations

$$
x(t+1)=A(t) x(t)+g(t), \quad t \in \mathbb{Z}_{+}
$$

where $g: \mathbb{Z}_{+} \rightarrow \mathbb{R}^{k}$ is almost periodic.

Let $l^{\infty}\left(\mathbb{Z}_{+}\right)$denote the Banach space of all bounded $\mathbb{R}^{k}$-valued sequences equipped with the sup norm defined for each $x=\{x(t)\}_{t \in \mathbb{Z}_{+}} \in l^{\infty}\left(\mathbb{Z}_{+}\right)$, by

$$
\|x\|_{\infty}=\sup _{t \in \mathbb{Z}_{+}}\|x(t)\| .
$$

Let $N\left(\mathbb{Z}_{+}\right) \subset l^{\infty}\left(\mathbb{Z}_{+}\right)$denote the subspace of all null sequences.

Definition 2.1. $[18,19,25,33,43,44]$ An $\mathbb{R}^{k}$-valued sequence $x=\{x(t)\}_{t \in \mathbb{Z}_{+}}$is called Bohr almost periodic if for each $\varepsilon>0$, there exists a positive integer $T_{0}(\varepsilon)$ such that among any $T_{0}(\varepsilon)$ consecutive integers, there exists at least one integer $\tau$ with the following property:

$$
\|x(t+\tau)-x(t)\|<\varepsilon, \forall t \in \mathbb{Z}_{+} .
$$

The integer $\tau$ is then called an $\varepsilon$-period of the sequence $x=\{x(t)\}_{t \in \mathbb{Z}_{+}}$. 
Definition 2.2. $[18,19,25,33,43]$ An $\mathbb{R}^{k}$-valuedsequence $x=\{x(t)\}_{t \in \mathbb{Z}_{+}}$is called Bochner almost periodic if for every sequence $\{h(t)\}_{t \in \mathbb{Z}_{+}} \subset \mathbb{Z}_{+}$there exists a subsequence $\left\{h\left(K_{s}\right)\right\}_{s \in \mathbb{Z}_{+}}$such that $\left\{x\left(t+h\left(K_{s}\right)\right)\right\}_{s \in \mathbb{Z}_{+}}$converges uniformly in $t \in \mathbb{Z}_{+}$.

One should point out that combining the extension theorem [2, Proposition 4.7 .1 , p. 305] (of an almost periodic function on $\mathbb{R}_{+}$to an almost periodic function $\mathbb{R})$ and $\left[6\right.$, Theorem 1.27 , p. 47], it is not hard to see that if $\{x(t)\}_{t \in \mathbb{Z}_{+}}$is an $\mathbb{R}^{k}$-valued almost periodic sequence, then there exists a unique almost periodic function $f: \mathbb{R} \mapsto \mathbb{R}^{k}$ such that $x(t)=f(t)$ for each $t \in \mathbb{Z}_{+}$. Unlike the case of almost periodicity on $\mathbb{Z}$, here, we make use of other techniques to establish our preliminary results on almost periodicity on $\mathbb{Z}_{+}$rather than equations of the form $x(t)=f(t), t \in \mathbb{Z}_{+}$.

We use the following result to reconcile the two definitions of almost periodic sequences.

Proposition 2.3. Let $x^{m}=\left\{x^{m}(t)\right\}_{t \in \mathbb{Z}_{+}}$be a Bohr almost periodic sequence converging uniformly in $m \in \mathbb{Z}_{+}$to $x$, then the sequence $x$ is Bohr almost periodic.

Proof. The proof is similar to the one in Halanay [25, Proposition 4.7, p. 229] for sequences in $\mathbb{Z}$ and hence omitted.

The next step consists of showing that the two definitions of almost periodic sequences in $\mathbb{Z}_{+}$are equivalent.

Theorem 2.4. A sequence $x=\{x(t)\}_{t \in \mathbb{Z}_{+}}$is Bochner almost periodic if and only if it is Bohr almost periodic.

Proof. First, we show that if $x=\{x(t)\}_{t \in \mathbb{Z}_{+}}$is Bochner almost periodic, then it is Bohr almost periodic. To achieve this, we show that if $x=\{x(t)\}_{t \in \mathbb{Z}_{+}}$is not Bohr almost periodic, then it is not Bochner almost periodic.

Suppose that $x=\{x(t)\}_{t \in \mathbb{Z}_{+}}$is not Bohr almost periodic. Then there exists at least one $\varepsilon>0$ such that for any positive integer $T_{0}$, there exist $T_{0}$ consecutive positive integers which contain no $\varepsilon$-period related to the sequence $\{x(t)\}_{t \in \mathbb{Z}_{+}}$. Now, let $h(1) \in \mathbb{Z}_{+}$and let $2 \alpha_{1}+1,2 \alpha_{1}+2,2 \alpha_{1}+3, \ldots, 2 \beta_{1}-2,2 \beta_{1}-1$ be $\left(2 \beta_{1}-2 \alpha_{1}-1\right)$ positive integers $\left(\alpha_{1}, \beta_{1} \in \mathbb{Z}_{+}\right)$such that $2 \beta_{1}-2 \alpha_{1}-2>2 h(1)$ or $\beta_{1}-\alpha_{1}-1>h(1)$ and the sequence $2 \alpha_{1}+1,2 \alpha_{1}+2,2 \alpha_{1}+3, \ldots, 2 \beta_{1}-2,2 \beta_{1}-1$ does not contain any $\varepsilon$-period related to $\{x(t)\}_{t \in \mathbb{Z}_{+}}$.

Next, let $h(2)=\frac{1}{2}\left(2 \alpha_{1}+2 \beta_{1}\right)=\alpha_{1}+\beta_{1}$. Clearly, $h(2)-h(1)$ is a (positive) integer such that $2 \alpha_{1}+1<h(2)-h(1)<2 \beta_{1}-1$, and hence $h(2)-h(1)$ cannot be an $\varepsilon$-period. Thus, there exist $2 \alpha_{2}+1,2 \alpha_{2}+2,2 \alpha_{2}+3, \ldots, 2 \beta_{2}-2,2 \beta_{2}-1$ such that $2 \beta_{2}-2 \alpha_{2}-2>2(h(1)+h(2))$, which does not contain any $\varepsilon$-period related to $\{x(t)\}_{t \in \mathbb{Z}_{+}}$. Setting $h(3)=\frac{1}{2}\left(2 \alpha_{2}+2 \beta_{2}\right)=\alpha_{2}+\beta_{2}$, it follows that $h(3)-h(2)$, $h(3)-h(1)$ are respectively one of the terms $2 \alpha_{2}+1,2 \alpha_{2}+2,2 \alpha_{2}+3, \ldots, 2 \beta_{2}-$ $2,2 \beta_{2}-1$, and hence $h(3)-h(2), h(3)-h(1)$ are not $\varepsilon$-period related to $\{x(t)\}_{t \in \mathbb{Z}_{+}}$. Proceeding as previously, one defines the numbers $h(4), h(5), \ldots$, such that none of the expressions $h(i)-h(j)$ for $i>j$ is an $\varepsilon$-period for the sequence $\{x(t)\}_{t \in \mathbb{Z}_{+}}$. 
Consequently, for all $i, j \in \mathbb{Z}_{+}$,

$$
\begin{aligned}
\sup _{i, j}\|x(t+h(i))-x(t+h(j))\| & \geq \sup _{i>j}\|x(t+h(i))-x(t+h(j))\| \\
& =\sup _{i>j}\|x(t+h(i)-h(j))-x(t)\| \\
& \geq \varepsilon .
\end{aligned}
$$

This proves that $\{x(t+h(i))\}_{i \in \mathbb{Z}_{+}}$cannot contain any uniformly convergent sequence, and hence $\{x(t)\}_{t \in \mathbb{Z}_{+}}$is not Bochner almost periodic.

Conversely, suppose that the sequence $\{x(t)\}_{t \in \mathbb{Z}_{+}}$is Bohr almost periodic and $\left\{t_{j}\right\}_{j \in \mathbb{Z}_{+}}$is a sequence of positive integers. Here, we adapt our proof to the one given in [25, Proof of Theorem 4.9., p. 230-231]. For each $\varepsilon>0$ there exists an integer $T_{0}>0$ such that between $t_{j}$ and $T_{0}+t_{j}$ there exist an $\varepsilon$-period $\tau_{j}$ with $0 \leq \tau_{j}-t_{j} \leq T_{0}$. Setting $s_{j}=\tau_{j}-t_{j}$, one can see that $s_{j}$ can take only a finite number (at most $T_{0}+1$ ) values, and hence there is some $s, 0 \leq s \leq T_{0}$ such that $s_{j}=s$ for an infinite numbers of $j^{\prime} s$. Let these indexes be numbered as $j_{k}$, then we have

Hence,

$$
\left\|x\left(t+t_{j}\right)-x\left(t+s_{j}\right)\right\|=\left\|x\left(t+\tau_{j}+s_{j}\right)-x\left(t+s_{j}\right)\right\|<\varepsilon
$$

for all $t \in \mathbb{Z}_{+}$.

$$
\left\|x\left(t+t_{j}\right)-x\left(t+s_{j}\right)\right\|<\varepsilon
$$

One may complete the proof by proceeding exactly as in [25, Proof of Theorem 4.9., pp. 230-231] and using [25, Proposition 4.7] relative to $\mathbb{Z}_{+}$rather than $\mathbb{Z}$.

Now let $\left\{\varepsilon_{r}\right\}_{r \in \mathbb{Z}_{+}}$be a sequence such that $\varepsilon \rightarrow 0$ as $r \rightarrow \infty$, say $\varepsilon_{r}=\frac{1}{r+1}$. Now, from the sequence $\left\{x\left(n+t_{j}\right)\right\}_{j \in \mathbb{Z}_{+}}$, consider a subsequence chosen so that

$$
\left\|x\left(n+t_{j_{i}^{1}}\right)-x\left(n+s^{1}\right)\right\| \leq \varepsilon_{1} .
$$

Next, from the previous sequence, we take a new subsequence such that

$$
\left\|x\left(n+t_{j_{i}^{2}}\right)-x\left(n+s^{2}\right)\right\| \leq \varepsilon_{2} .
$$

Repeating this procedure and for each $r \in Z_{+}$we obtain a subsequence $\left\{x\left(n+t_{j_{i}^{r}}\right)\right\}_{i \in \mathbb{Z}_{+}}$ such that

$$
\left\|x\left(n+t_{j_{i}^{r}}\right)-x\left(n+s^{r}\right)\right\| \leq \varepsilon_{r} .
$$

Now, for the diagonal sequence, $\left\{x\left(n+t_{j_{i}^{i}}\right)\right\}_{i \in \mathbb{Z}_{+}}$, for each $\varepsilon>0$ take $k(\varepsilon) \in \mathbb{Z}_{+}$ such that $\varepsilon_{k(\varepsilon)}<\frac{\varepsilon}{2}$, where $\varepsilon_{r}$ belongs to the previous sequence $\left\{\varepsilon_{r}\right\}_{r \in \mathbb{Z}_{+}}$.

Using the fact that the sequences $\left\{t_{j_{r}^{r}}\right\}$ and $\left\{t_{j_{s}^{s}}\right\}$ are both subsequences of $\left\{t_{j_{i}^{k(\varepsilon)}}\right\}$, for $r \geq k(\varepsilon)$ we have

$$
\begin{aligned}
\left\|x\left(n+t_{j_{r}^{r}}\right)-x\left(n+t_{j_{s}^{s}}\right)\right\| & \leq\left\|x\left(n+t_{j_{r}^{r}}\right)-x\left(n+s^{k}\right)\right\| \\
& +\left\|x\left(n+s^{k}\right)-x\left(n+t_{j_{s}^{s}}\right)\right\| \\
& \leq \varepsilon_{k(\varepsilon)}+\varepsilon_{k(\varepsilon)} \\
& \leq \varepsilon .
\end{aligned}
$$

Thus, the sequence $\left\{x\left(n+t_{j_{i}^{i}}\right)\right\}_{i \in \mathbb{Z}_{+}}$is a Cauchy sequence. 
Let $x=\{x(i)\}_{i \in \mathbb{Z}_{+}}$and $\alpha=\{\alpha(j)\}_{j \in \mathbb{Z}_{+}}$be $\mathbb{R}^{k}$-valued sequences. Define

$$
T_{\alpha} x:=\left\{y=\left(y_{j}\right)_{j \in \mathbb{Z}_{+}}: y(j)=\lim _{i \rightarrow \infty} x(j+\alpha(i))\right\} .
$$

Theorem 2.5. Let $x$ be a sequence. Suppose that for every pair $\alpha^{\prime}, \beta^{\prime}$ of sequences in $\mathbb{Z}_{+}$there exist common subsequences $\alpha, \beta$ where $\alpha$ is a subsequence of $\alpha^{\prime}$ and $\beta$ that of $\beta^{\prime}$, such that $T_{\alpha} T_{\beta} x=T_{\alpha+\beta} x$ pointwise in $\mathbb{Z}_{+}$. Then $x$ is almost periodic.

The proof of Theorem 2.5 is similar to the one given for sequences in $\mathbb{Z}[25$, Theorem 4.18, pp 234-235], and is omitted.

The collection of all almost periodic $\mathbb{R}^{k}$-valued sequences on $\mathbb{Z}_{+}$will be denoted by $A P\left(\mathbb{Z}_{+}\right)$. It is a Banach space when equipped with the sup norm defined above.

Lemma 2.6. If $\{x(t)\}_{t \in \mathbb{Z}_{+}}$is almost periodic, then it is bounded.

Proof. Assume that $\{x(t)\}_{t \in \mathbb{Z}_{+}}$is not bounded. Then for some subsequence

$$
\left\|x\left(t_{i}\right)\right\| \rightarrow \infty \text { as } i \rightarrow \infty \text {. }
$$

Let $\varepsilon=1$. Then there exists $T(\varepsilon) \in \mathbb{Z}_{+}-\{0\}$ that satisfies the almost periodicity definition. There exists $t_{i}=s_{1}$ such that $t_{i}=s_{1}>T(\varepsilon)$. Then among the integers

$$
\left\{s_{1}-T(\varepsilon)+1, s_{1}-T(\varepsilon)+2, \ldots, s_{1}\right\}
$$

there exists $\widehat{s}_{1}$ such that

$$
\left\|x\left(t+\widehat{s}_{1}\right)-x(t)\right\|<1 .
$$

Next, choose $t_{j}=s_{2}$ such that $t_{j}=s_{2}>T(\varepsilon)+s_{1}$. Then among the integers

$$
\left\{s_{2}-T(\varepsilon)+1, s_{2}-T(\varepsilon)+2, \ldots, s_{2}\right\}
$$

there exists $\widehat{s}_{2}$ such that

$$
\left\|x\left(t+\widehat{s}_{2}\right)-x(t)\right\|<1 .
$$

Repeating this process, we obtain a sequence $\left\{\widehat{s}_{i}\right\} \rightarrow \infty$ as $i \rightarrow \infty$ such that

$$
\left\|x\left(t+\widehat{s}_{i}\right)-x(t)\right\|<1 \text { for } r=1,2,3, \ldots,
$$

and a subsequence $\left\{s_{i}\right\}$ of $\left\{t_{i}\right\}$ with $\left\{s_{i}\right\} \rightarrow \infty$ as $i \rightarrow \infty$. Moreover,

$$
s_{i}=\widehat{s}_{i}+u_{i}
$$

where $0 \leq u_{i}<T(\varepsilon)$.

Since $\left\{u_{i}\right\}$ is finite, there exists $u_{i_{0}}$ that is repeated infinitely many times and $s_{i_{r}}=\widehat{s}_{i_{r}}+u_{i_{0}}$, where $i_{r} \rightarrow \infty$ as $i \rightarrow \infty$. Therefore,

$$
\left\|x\left(t+\widehat{s}_{i_{r}}\right)-x\left(u_{i_{0}}\right)\right\|<1 \text {. }
$$

Moreover,

$$
\left\|x\left(t+s_{i_{r}}\right)-x\left(u_{i_{0}}\right)\right\|<1 .
$$

Hence, $\left\{x\left(s_{i_{r}}\right)\right\}$ is bounded; a contradiction.

Definition 2.7. A real-valued sequence $x=\{x(t)\}_{t \in \mathbb{Z}_{+}}$is said to be asymptotically almost periodic if it can be decomposed as

$$
x(t)=u(t)+v(t),
$$

where $u=\{u(t)\}_{t \in \mathbb{Z}_{+}} \in A P\left(\mathbb{Z}_{+}\right)$and $\{v(t)\}_{t \in \mathbb{Z}_{+}} \in N\left(\mathbb{Z}_{+}\right)$.

The collection of all asymptotically almost periodic $\mathbb{R}^{k}$-valued sequences will be denoted by $A A P\left(\mathbb{Z}_{+}\right)$. 
Definitions 2.1, 2.2 and 2.7 are respectively adapted from the definitions of $\mathbb{R}^{k}$ valued almost periodic sequences and asymptotically almost periodic sequences $x=\{x(t)\}_{t \in \mathbb{Z}}$ defined in $\mathbb{Z}$.

Lemma 2.8. If $x=\{x(t)\}_{t \in \mathbb{Z}_{+}} \in A P\left(\mathbb{Z}_{+}\right)$and $\lim _{t \rightarrow \infty} x(t)=0$, then $x(t)=0$ for all $t \in \mathbb{Z}_{+}$.

Proof. Let $\varepsilon_{t}=\frac{1}{t+1}$ for each $t \in\{0,1, \ldots\}$. Then there exists $T\left(\varepsilon_{t}\right)$ such that among $t, t+1, \ldots, t+T\left(\varepsilon_{t}\right)-1$, there exists $s_{t}$ such that

$$
\left\|x\left(t+s_{t}\right)-x(t)\right\| \leq \varepsilon_{t} \text { for all } t \in \mathbb{Z}_{+} .
$$

As $t \rightarrow \infty, s_{t} \rightarrow \infty, x\left(t+s_{t}\right) \rightarrow 0$, and $\varepsilon_{t} \rightarrow 0$. Hence, $\|x(t)\| \leq 0$. This implies that $x(t)=0$ for all $t \in \mathbb{Z}_{+}$.

Lemma 2.9. The decomposition of an asymptotically almost periodic sequence is unique. That is, $A P\left(\mathbb{Z}_{+}\right) \cap N\left(\mathbb{Z}_{+}\right)=\{0\}$.

Proof. Suppose that $x=\{x(t)\}_{t \in \mathbb{Z}_{+}}$can be decomposed as

$$
x(t)=u(t)+v(t)
$$

and

$$
x(t)=v(t)+\delta(t),
$$

where $u=\{u(t)\}_{t \in \mathbb{Z}_{+}}, \nu=\{\nu(t)\}_{t \in \mathbb{Z}_{+}} \in A P\left(\mathbb{Z}_{+}\right)$and $\{v(t)\}_{t \in \mathbb{Z}_{+}},\{\delta(t)\}_{t \in \mathbb{Z}_{+}} \in$ $N\left(\mathbb{Z}_{+}\right)$. Clearly, $u(t)-\nu(t)=\delta(t)-v(t) \in A P\left(\mathbb{Z}_{+}\right) \cap N\left(\mathbb{Z}_{+}\right)$. By Lemma 2.8, $u(t)=\nu(t)$ and $v(t)=\delta(t)$ for all $t \in \mathbb{Z}_{+}$.

Theorem 2.10. Assume that $(t, w) \mapsto f(t, w)$ is Lipschitz in $w$ uniformly in $t \in$ $\mathbb{Z}^{+}$. If $x(t)=u(t)+v(t)$ is a solution of $E q$. (2.1), then $\{u(t)\}_{t \in \mathbb{Z}_{+}}$is also a solution of Eq. (2.1), where $u=\{u(t)\}_{t \in \mathbb{Z}_{+}} \in A P\left(\mathbb{Z}_{+}\right)$and $\nu=\{\nu(t)\}_{t \in \mathbb{Z}_{+}} \in N\left(\mathbb{Z}_{+}\right)$.

Proof. Let $\{x(t)\}_{t \in \mathbb{Z}_{+}}$be an asymptotically almost periodic solution of Eq. (2.1). That is, $x(t)=u(t)+v(t)$, where $\{u(t)\}_{t \in \mathbb{Z}_{+}} \in A P\left(\mathbb{Z}_{+}\right)$and $\{v(t)\}_{t \in \mathbb{Z}_{+}} \in N\left(\mathbb{Z}_{+}\right)$.

Now

$$
\begin{aligned}
u(t+1)-A(t) u(t)-f(t, u(t)) & =x(t+1)-v(t+1)-A(t) u(t)-f(t, u(t)) \\
& =A(t) x(t)+f(t, x(t))-v(t+1) \\
& -A(t) u(t)-f(t, u(t)) .
\end{aligned}
$$

Consequently,

$$
\begin{aligned}
& \|u(t+1)-A(t) u(t)-f(t, u(t))\| \\
\leq \quad & (L+\|A(t)\|) \cdot \| x(t)-u(t))\|+\| v(t+1) \| \\
\leq & \left.\left(L+\sup _{t \in \mathbb{Z}_{+}}\|A(t)\|\right) \cdot \| v(t)\right)\|+\| v(t+1) \| .
\end{aligned}
$$

Hence, $\|u(t+1)-A(t) u(t)-f(t, u(t))\| \rightarrow 0$ as $t \rightarrow \infty$. 
Let $w(t)=u(t+1)-A(t) u(t)-f(t, u(t))$. If $w(T) \neq 0$ for some $t \in \mathbb{Z}_{+}$, let $\varepsilon=\frac{|w(T)|}{2}>0$. Thus,

$$
|w(T+p)-w(T)| \leq \frac{|w(T)|}{2}
$$

Hence,

$$
|w(T+p)| \geq \frac{|w(T)|}{2}
$$

Let $I_{t}=[s l,(s+1) l]$ be intervals of length $l$. For each interval $I_{s}$ there exists $p_{s}$ such that $\left|w\left(T+p_{s}\right)\right| \geq \frac{|w(T)|}{2}$. As $s \rightarrow \infty, p_{s} \rightarrow \infty$, and

$$
\lim _{t \rightarrow \infty}|w(t)| \geq \frac{|w(T)|}{2}>0 \text {. }
$$

This contradicts the fact that $\lim _{t \rightarrow \infty}|w(t)|=0$. Therefore, $w(t)=0$ for each $t \in \mathbb{Z}_{+}$. That is, $u(t+1)=A(t) u(t)+f(t, u(t))$ where $\{u(t)\}_{t \in \mathbb{Z}_{+}}$is almost periodic.

Definition 2.11. A sequence $F: \mathbb{Z}_{+} \times \mathbb{R}^{p} \mapsto \mathbb{R}^{q},(t, u) \mapsto F(t, u)$ is called almost periodic in $t \in \mathbb{Z}_{+}$uniformly in $u \in \mathbb{R}^{q}$ if for each $\varepsilon>0$ there exists a positive integer $T_{0}(\varepsilon)$ such that among any $T_{0}(\varepsilon)$ consecutive integers, there exists at least one integer $s$ with the following property:

$$
\|F(t+s, u)-F(t, u)\|<\varepsilon
$$

for all $u \in \mathbb{R}^{q}$ and $t \in \mathbb{Z}_{+}$.

Theorem 2.12. Suppose that $F: \mathbb{Z}_{+} \times \mathbb{R}^{p} \rightarrow \mathbb{R}^{q},(t, u) \mapsto F(t, u)$ is almost periodic in $t \in \mathbb{Z}_{+}$uniformly in $u \in \mathbb{R}^{p}$. If in addition, $F$ is Lipschitz in $u \in \mathbb{R}^{p}$ uniformly in $t \in \mathbb{Z}_{+}$(that is, there exists $L>0$ such that

$$
\left.\|F(t, u)-F(t, v)\| \leq L\|u-v\| \quad \forall u, v \in \mathbb{R}^{p}, t \in \mathbb{Z}_{+}\right),
$$

then for every $\mathbb{R}^{p}$-valued almost periodic sequence $x=\{x(t)\}_{t \in \mathbb{Z}^{+}}$, the $\mathbb{R}^{q}$-valued sequence $y(t)=F(t, x(t))$ is almost periodic.

Proof. Let $x=\{x(t)\}_{t \in \mathbb{Z}_{+}}$be an almost periodic sequence and let $y(t)=F(t, x(t))$. Then, for each $\varepsilon>0$ there exists a positive integer $T_{0}(\varepsilon)$ such that among any $T_{0}(\varepsilon)$ consecutive integers, there exists at least one integer $s$ with the following property:

Moreover,

$$
\|x(t+s)-x(t)\|<\frac{\varepsilon}{L} \quad \forall t \in \mathbb{Z}_{+} .
$$

$$
\begin{aligned}
\|y(t+s)-y(t)\| \leq & \|F(t+s, x(t+s))-F(t, x(t))\| \\
\leq & \|F(t+s, x(t+s))-F(t+s, x(t))\| \\
& +\|F(t+s, x(t))-F(t, x(t))\| \\
\leq & L \| x(t+s)-x(t)) \|+\frac{\varepsilon}{2} \\
< & \frac{\varepsilon}{2}+\frac{\varepsilon}{2}=\varepsilon .
\end{aligned}
$$




\section{Construction of Almost Periodic Sequences on $\mathbb{Z}_{+}$}

There are two ways of generating an almost periodic sequence on $\mathbb{Z}_{+}$. One may start with a periodic function on $\mathbb{R}$ or an almost periodic function on $\mathbb{R}$. In the sequel we will describe these two approaches.

(i) Periodic Functions. Let $f: \mathbb{R} \rightarrow \mathbb{R}^{k}$ be a periodic function with periodic $\omega$.

(a) If $\omega$ is a rational number, $\omega=\frac{r}{s}, r, s \in \mathbb{Z}_{+}$with $s \neq 0$, then $r=s \omega$ is also a period of $f$. If we let $x(t)=f(t), t \in \mathbb{Z}$, then $(x(t))_{t \in \mathbb{Z}_{+}}$is a periodic sequence of period $r$.

(b) Now assume that $\omega$ is an irrational number. Then $f$ is uniformly continuous and hence, given $\varepsilon>0$, there exists $\delta>0$ such that $|x-y|<\delta$ implies

$$
\|f(x)-f(y)\|<\varepsilon \text {. }
$$

There exists a rational number $\frac{r}{s}$ such that $\left|\omega-\frac{r}{s}\right|<\frac{\delta}{s}$ and consequently,

$$
|s \omega-r|<\delta .
$$

Define $x(t)=f(t), t \in \mathbb{Z}$. Then

$$
\begin{aligned}
\|x(t+r)-x(t)\| & \leq\|f(t+r)-f(t+s \omega)\| \\
& +\|f(t+s \omega)-f(t)\| \\
& <\varepsilon
\end{aligned}
$$

by Eq. (3.1).

(ii) Almost Periodic Functions. This case can also be divided into two cases. Let $f: \mathbb{R} \mapsto \mathbb{R}^{k}$ be an almost periodic function, and $\varepsilon>0$ be given. Then there exists $T(\varepsilon)$ such that for any interval of length $T(\varepsilon)$, there exists a number $\omega$ with

$$
\|f(t+\omega)-f(t)\|<\varepsilon \text { for all } t \in \mathbb{R} .
$$

(a) If $\omega=\frac{r}{s}$ is rational, then we consider $\tilde{T}(\varepsilon)=s T(\varepsilon), \tilde{\omega}=s \omega$. If we let $x(t)=f(t), t \in \mathbb{Z}$, it follows that $\tilde{T}(\varepsilon)$ and $\tilde{\omega}$ are the integers needed to make $x(t)$ almost periodic on $\mathbb{Z}$ and

$$
\|f(t+\tilde{\omega})-f(t)\|<\frac{\varepsilon}{2 s} .
$$

(b) Now assume that $\omega$ is irrational. Since $f$ is uniformly continuous, given $\varepsilon>0$, there exists $\delta>0$ such that $|x-y|<\delta$ implies $\|f(x)-f(y)\|<\frac{\varepsilon}{2}$. There exists a rational number $\frac{r}{s}, r, s \in \mathbb{Z}_{+}$with $s \neq 0$ such that $\left|\frac{r}{s}-\omega\right|<$ $\frac{\delta}{s}$. Hence $|r-s \omega|<\delta$ and consequently,

$$
\|f(t+r)-f(t+s \omega)\|<\frac{\varepsilon}{2}, \text { for all } t \in \mathbb{R} .
$$

Define $x(t)=f(t), t \in \mathbb{Z}$. Then

$$
\begin{aligned}
\|x(t+r)-x(t)\| & \leq\|f(t+r)-f(t+s \omega)\| \\
& +\|f(t+s \omega)-f(t)\| \\
& <\frac{\varepsilon}{2}
\end{aligned}
$$


by Eq. (3.3) and Eq. (3.4).

Let $T(\varepsilon)=\lfloor s \omega\rfloor+1(\lfloor\cdot\rfloor$ being the greatest integer function). Then $r$

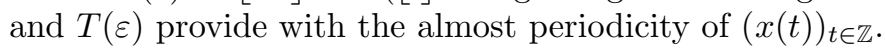

I. Finally, once we get an almost periodic sequence $\{x(t)\}_{t \in \mathbb{Z}}$, we define an associated sequence $\{\tilde{x}(t)\}$, where $\tilde{x}(t)=x(t) u_{0}(t)$ ( $u_{0}$ being the Heaviside function). The sequence $\{\tilde{x}(t)\}$ is almost periodic on $\mathbb{Z}_{+}$but not on $\mathbb{Z}$.

II. (a) An example for case (i) is the function $f(t)=\cos (\alpha t)$ with period $\omega=\frac{2 \pi}{\alpha}$. Then $\tilde{x}(t)=\cos (\alpha t) u_{0}(t)$ is almost periodic on $\mathbb{Z}_{+}$.

(b) For case (ii), we let $f(t)=\cos (\alpha t)+\cos (\beta t)$ with $\frac{2 \pi}{\alpha}$ or $\frac{2 \pi}{\beta}$ is irrational. Then $\tilde{x}(t)=(\cos (\alpha t)+\sin (\beta t)) u_{0}(t)$ is almost periodic on $\mathbb{Z}_{+}$but not on $\mathbb{Z}$.

Remark 3.1. (i) Since almost periodic functions $f$ on $\mathbb{R}$ can be approximated by trigonometric polynomials, it follows that almost periodic sequences $\tilde{x}(t)$ on $\mathbb{Z}_{+}$ that are constructed in the manner described above can also be approximated by trigonometric sequences.

(ii) Almost periodic sequences may be generated as solutions of scalar difference equations or systems of difference equations. The following examples elucidate this point.

Example 3.2. Consider the second-order difference equation

$$
x(t+2)-2 \cos \alpha u_{0}(t) x(t+1)+x(t)=0, \quad x(0)=0, x(1)=1,
$$

where $0<\alpha<\pi$ and $\alpha$ is not a multiple of $\pi$. Then the solution is given by

$$
x(t)= \begin{cases}\frac{\sin (t \alpha)}{\sin \alpha} & \text { if } t \geq 0 \\ \ldots \overline{x(-4), x(-3), x(-2), x(-1)} & \text { if } t<0\end{cases}
$$

where $x(-1)=-1, x(-2)=0, x(-3)=1, x(-4)=0$, and this is of period 4 .

Clearly, $\{x(t)\}$ is almost periodic on $\mathbb{Z}_{+}$but not on $\mathbb{Z}$.

Example 3.3. This set of examples is inspired by Corduneanu [6, Theorem 8]. Consider the $k$-dimensional system of difference equations

$$
x(t+1)=A x(t)+g(t)
$$

where $A$ is a $k \times k$-matrix and $g$ is assumed to be almost periodic on $\mathbb{Z}$. Then by [6, Theorem 8], any bounded solution of the previous system is necessarily almost periodic on $\mathbb{Z}$. And by the construction above this produces an almost periodic sequence on $\mathbb{Z}_{+}$.

\section{Regular Exponential Dichotomy}

In Eq. (2.2), the classical definition of dichotomy does not apply, whenever the state transition matrix

$$
X(t, s)=\prod_{r=s}^{t-1} A(r)
$$

is not invertible. In [27], Henry used the following definition to address this problem. 
Definition 4.1. Eq. (2.2) is said to have a regular exponential dichotomy if there exists $k \times k$ projection matrices $P(t)$ with $t \in \mathbb{Z}_{+}$and positive constants $M$ and $\beta \in(0,1)$ such that the following four conditions are satisfied:

(i) $\quad A(t) P(t)=P(t+1) A(t)$;

(ii) The matrix $\left.A(t)\right|_{R(I-P(t))}$ is an isomorphism from $R(I-P(t))$ onto $R(I-$ $P(t+1))$

(iii) $\|X(t, r) P(r) x\| \leq M \beta^{t-r}\|x\|$, for $0 \leq r \leq t, x \in \mathbb{R}^{k}$;

(iv) $\|X(r, t)(I-P(t)) x\| \leq M \beta^{t-r}\|x\|$, for $0 \leq r \leq t, x \in \mathbb{R}^{k}$.

By repeated application of [(i), Definition 4.1], we obtain

$$
P(t) X(t, s)=X(t, s) P(s) .
$$

Define the hull $H(x)$ of a sequence $x$ as follows:

Definition 4.2. The set

$$
H(x)=\left\{\widetilde{x} \mid \text { there exists a sequence } \alpha \text { in } \mathbb{Z}_{+} \text {with } T_{\alpha} x=\widetilde{x}\right\} .
$$

Similarly, for a matrix function $A(t)$, we define

$$
H(A)=\left\{\widetilde{A} \mid \text { there exists a sequence } \alpha \text { in } \mathbb{Z}_{+} \text {with } T_{\alpha} A=\widetilde{A}\right\},
$$

where $T_{\alpha} A=\widetilde{A}$ means that $\lim _{t \rightarrow \infty} A(t+\alpha(t))=\widetilde{A}(t)$.

Theorem 4.3. Suppose that Eq. (2.2) has a regular exponential dichotomy and $\widetilde{A}(t) \in H(A(t))$. Then the system

$$
x(t+1)=\widetilde{A}(t) x(t)
$$

satisfies a regular exponential dichotomy with same projections and constants.

Proof. Let $T_{\alpha} A=\widetilde{A}$. Then $X_{i}(t)=X\left(t+\alpha_{i}\right)$ is a fundamental matrix for the equation

$$
x(t+1)=A\left(t+\alpha_{i}\right) x(t)
$$

and satisfies regular exponential dichotomy with the same projection $P(i)$ and same constants $M$ and $\beta$.

One may take subsequences so that $X_{i}(0)$ converges to $Y_{0}$. For a suitable subsequence, $X_{i}(t)$ converges to a solution $Y(t)$ of

$$
x(t+1)=\widetilde{A}(t) x(t) .
$$

Then $Y(0)=Y_{0}$ and $Y(t)$ satisfies the conditions of regular exponential dichotomy.

Theorem 4.4. Suppose that Eq. (2.2) has a regular exponential dichotomy and $\widetilde{A}(t) \in H(A(t))$. Then, Eq. (2.3) has an almost periodic solution given by

$$
\bar{x}(t)=\sum_{r=-\infty}^{t-1} X(t, r+1) P(r+1) g(r)-\sum_{r=t}^{\infty} X(t, r+1)(I-P(r+1)) g(r),
$$

where $X(t, r) P(r)=0$ for $r>t$ and $g(r)=0$ for $r<0$. 
Proof. It can be easily verified that $\bar{x}(t)$ defined by Eq. (4.2) is indeed a solution of Eq. (2.3). Moreover,

$$
\begin{aligned}
\|\bar{x}(t)\| & \leq\left\{\sum_{r=-\infty}^{t-1}\|X(t, r+1) P(r+1)\|+\sum_{r=t}^{\infty}\|X(t, r+1)(I-P(r+1))\|\right\}\|g\| \\
& \leq\left\{\sum_{r=0}^{t-1} M \beta^{t-r-1}+\sum_{r=t}^{\infty} M \beta^{r+1-t}\right\}\|g\| \leq M \frac{1+\beta}{1-\beta}\|g\| .
\end{aligned}
$$

Let $\{\widetilde{\alpha}\}$ and $\{\widetilde{\gamma}\}$ be arbitrary sequences of nonnegative integers, and let $\{\alpha\} \subset$ $\{\widetilde{\alpha}\}$ and $\{\gamma\} \subset\{\widetilde{\gamma}\}$ be their common subsequences. Then $T_{\alpha+\gamma} A=T_{\gamma} T_{\alpha} A$ and $T_{\alpha+\gamma} g=T_{\gamma} T_{\alpha} g$. Now,

$$
\begin{aligned}
& \bar{x}\left(t+\alpha_{i}\right)=\sum_{r=-\infty}^{t+\alpha_{i}-1} X\left(t+\alpha_{i}, r+1\right) P(r+1) g(r)- \\
& \sum_{r=t+\alpha_{i}}^{\infty} X\left(t+\alpha_{i}, r+1\right)(I-P(r+1)) g(r) \\
& =\sum_{s=-\infty}^{t-1} X\left(t+\alpha_{i}, s+\alpha_{i}+1\right) P\left(s+\alpha_{i}+1\right) g\left(s+\alpha_{i}\right)- \\
& \sum_{s=t}^{\infty} X\left(t+\alpha_{i}, s+\alpha_{i}+1\right)\left(I-P\left(s+\alpha_{i}+1\right)\right) g\left(s+\alpha_{i}\right) \\
& =\sum_{s=-\infty}^{t-1} A\left(t+\alpha_{i}-1\right) \cdots A\left(s+\alpha_{i}+1\right) P\left(s+\alpha_{i}+1\right) g\left(s+\alpha_{i}\right)- \\
& \sum_{s=t}^{\infty} A\left(t+\alpha_{i}-1\right) \cdots A\left(s+\alpha_{i}+1\right)\left(I-P\left(s+\alpha_{i}+1\right)\right) g\left(s+\alpha_{i}\right) . \\
& \lim _{i \rightarrow \infty} \bar{x}\left(t+\alpha_{i}\right)=\left(T_{\alpha} \bar{x}\right)_{t}=\sum_{s=-\infty}^{t-1} \widetilde{A}(t-1) \cdots \widetilde{A}(s+1) \widetilde{P}(s+1) \widetilde{g}(s)- \\
& \sum_{s=t}^{\infty} \widetilde{A}(t-1) \cdots \widetilde{A}(s+1)(I-\widetilde{P}(s+1)) \widetilde{g}(s) \\
& =\sum_{s=-\infty}^{t-1}\left(T_{\alpha} A\right)_{t-1} \cdots\left(T_{\alpha} A\right)_{s+1}\left(T_{\alpha} P\right)_{s+1}\left(T_{\alpha} g\right)_{s}- \\
& \sum_{s=t}^{\infty}\left(T_{\alpha} A\right)_{t-1} \cdots\left(T_{\alpha} A\right)_{s+1}\left(I-T_{\alpha} P\right)_{s+1}\left(T_{\alpha} g\right)_{s} .
\end{aligned}
$$

Moreover,

$$
\begin{aligned}
\left(T_{\gamma} T_{\alpha} \bar{x}\right)_{t}= & \sum_{s=-\infty}^{t-1}\left(T_{\gamma} T_{\alpha} A\right)_{t-1} \cdots\left(T_{\gamma} T_{\alpha} A\right)_{s+1}\left(T_{\gamma} T_{\alpha} P\right)_{s+1}\left(T_{\gamma} T_{\alpha} g\right)_{s}- \\
& \sum_{s=t}^{t-1}\left(T_{\gamma} T_{\alpha} A\right)_{t-1} \cdots\left(T_{\gamma} T_{\alpha} A\right)_{s+1}\left(I-T_{\gamma} T_{\alpha} P\right)_{s+1}\left(T_{\gamma} T_{\alpha} g\right)_{s} \\
= & \left(T_{\gamma+\alpha} \bar{x}\right)_{t} .
\end{aligned}
$$


Hence, $\{\bar{x}(t)\}_{t \in \mathbb{Z}_{+}} \in A P\left(\mathbb{Z}_{+}\right)$.

Corollary 4.5. If the zero solution of Eq. (2.2) is uniformly asymptotically stable, then Eq. (2.3) has a unique globally asymptotically stable almost periodic solution,

$$
\bar{x}(t)=\sum_{r=0}^{t-1}\left(\prod_{s=r}^{t-1} A(s)\right) g(r) .
$$

Moreover,

$$
\|\bar{x}(t)\| \leq \frac{M \beta}{1-\beta}\|g\| .
$$

Proof. Let $y(t)$ be a solution of Eq. (2.3) with $y(0)=y_{0}$. Then,

$$
y(t)=X(t) y_{0}+\sum_{r=0}^{t-1}\left(\prod_{s=r}^{t-1} A(s)\right) g(r) .
$$

Therefore,

$$
y(t)=\gamma(t)+\bar{x}(t)
$$

where $\gamma(t)$ is a null sequence. Thus, $y(t)$ is an asymptotically almost periodic solution of Eq. (2.3). By Lemma 2.9, $y(t) \in A P\left(\mathbb{Z}_{+}\right)$implies that $y=\bar{x}$. Hence,

$$
\bar{x}(t)=\sum_{r=0}^{t-1}\left(\prod_{s=r}^{t-1} A(s)\right) g(r)
$$

is the only almost periodic solution of Eq. (2.3).

It is easy to see that $\|\bar{x}(t)\| \leq \frac{M \beta}{1-\beta}\|g\|$.

Theorem 4.6. Suppose that $f$ is Lipschitz with Lipschitz constant L. Then Eq. (2.1) has a unique globally asymptotically stable almost periodic solution if

$$
\frac{M \beta L}{1-\beta}<1 .
$$

Proof. Consider the Banach space $A P\left(\mathbb{Z}_{+}\right)$equipped with the sup norm. By Theorem 2.12 , if $\varphi \in A P\left(\mathbb{Z}_{+}\right)$then $f(t, \varphi(t)) \in A P\left(\mathbb{Z}_{+}\right)$. Let

$$
\Gamma: A P\left(\mathbb{Z}_{+}\right) \rightarrow A P\left(\mathbb{Z}_{+}\right)
$$

be the nonlinear operator defined by

$$
(\Gamma \varphi)(t):=\sum_{r=0}^{t-1}\left(\prod_{s=r}^{t-1} A(s)\right) f(r, \varphi(r)) .
$$

By Theorem 4.4, $\Gamma$ is well defined. Moreover, for $\varphi, \psi \in A P\left(\mathbb{Z}_{+}\right)$,

$$
\|(\Gamma \varphi)(t)-(\Gamma \psi)(t)\| \leq \frac{M \beta}{1-\beta}\|f(t, \varphi(t))-f(t, \psi(t))\|,
$$

and

$$
\|\Gamma \varphi-\Gamma \psi\|_{\infty} \leq \frac{M \beta L}{1-\beta}\|\varphi-\psi\|_{\infty}
$$


$\Gamma$ is a contraction whenever $\frac{M \beta L}{1-\beta}<1$. Using the Banach fixed point theorem, we obtain that $\Gamma$ has a unique fixed point, $\bar{x}$. Moreover, $\bar{x}$ is the globally asymptotically stable almost periodic solution of Eq. (2.1).

\section{Population Models With Non-Overlapping Generations}

In some plant populations, growth is a discrete process and generations do not overlap. To study the impact of periodic environments on the long-term population dynamics of such populations, various researchers have used simple models of the general form

$$
x(t+1)=f(t, x(t)), \quad t \in \mathbb{Z}_{+} .
$$

where $x(t)$ is the population size at generation $t$ [13-16, 20-23]. The smooth map $f: \mathbb{Z}^{+} \times[0, \infty) \rightarrow(0, \infty)$ is the per capita growth rate.

In periodic environments, $f$ is periodic with period $p$. That is, there exists a smallest positive integer $p$ satisfying $f(t+p, x)=f(t, x)$.

The periodic Beverton-Holt model,

$$
x(t+1)=\frac{\mu K_{t} x(t)}{K_{t}+(\mu-1) x(t)},
$$

where the constant intrinsic growth rate $\mu>1$ and the carrying capacity $K_{t}$ is periodic with minimal period $p \geq 2$,

$$
K_{t+p}=K_{t}>0
$$

is an example of Eq. (5.2) in periodic environments.

Eq. (2.1) with the linear part, $A(t) x(t)$, missing reduces to Eq. (5.1). In this case, one may contemplate the variational system

$$
y(t+1)=B(t) y(t)+g(t, y(t)), \quad t \in \mathbb{Z}_{+}
$$

where

$$
B(t)=\frac{\partial}{\partial x} f(t, z(t))
$$

for some solution $z(t)$ of Eq. (5.1), and

$$
g(t, y(t))=f(t, y(t))-B(t) y(t) .
$$

However, this approach has at least two basic problems. The first problem is that, it is difficult to find the solution $z(t)$ of Eq. (5.1). The second problem is that, the linear part of Eq. (5)) does not satisfy the hypotheses of Theorem 4.6.

To illustrate the difficulties in a specific example, we consider the almost periodically forced Beverton-Holt model. That is, in Eq. (5.2) we assume that $\left\{K_{t}\right\}_{t \in \mathbb{Z}_{+}}$ is almost periodic in $\mathbb{Z}_{+}$and $\mu>1$. The variational equation around $z(t)=0$ associated with Eq. (5.2) is given by

$$
x(t+1)=\mu x(t)-\frac{\mu(\mu-1)(x(t))^{2}}{K_{t}+(\mu-1) x(t)} .
$$

However, $\mu>1$ implies that Theorem 4.6 does not apply.

A simple substitution transforms Eq. (5.2) into a linear equation. To be more specific,

$$
y(t)=\frac{1}{x(t)}
$$


in Eq. (5.2) yields

$$
y(t+1)=\frac{1}{\mu} y(t)+\frac{\mu-1}{\mu K_{t}}, \quad t \in \mathbb{Z}_{+}
$$

Since $K_{t} \in A P\left(\mathbb{Z}_{+}\right)$, we have $\frac{\mu-1}{\mu K_{t}} \in A P\left(\mathbb{Z}_{+}\right)$. Hence, Corollary 4.5 applies and Eq. (5.3) has a unique globally asymptotically stable almost periodic solution $\overline{y(t)}$. Consequently,

$$
\overline{x(t)}=\frac{1}{\overline{y(t)}}
$$

is the unique almost periodic solution of Eq. (5.2), where $K_{t} \in A P\left(\mathbb{Z}_{+}\right)$and $\mu>1$. Moreover, for any solution $x(t)$ of Eq. (5.2) we have

$$
|x(t)-\overline{x(t)}|=\left|\frac{1}{y(t)}-\frac{1}{\overline{y(t)}}\right|=\frac{|y(t)-\overline{y(t)}|}{|y(t) \overline{y(t)}|} .
$$

So both $y(t)$ and $\overline{y(t)}$ are bounded away from zero. As a result,

$$
|x(t)-\overline{x(t)}| \leq M|y(t)-\overline{y(t)}| \text {. }
$$

Hence, $\overline{x(t)}$ is globally asymptotically stable. This result was obtained in [32] under the assumption that the sequence $\left\{K_{t}\right\}$ is defined for all $t \in \mathbb{Z}_{+}$.

\section{Population Models With Overlapping Generations}

In constant environments, theoretical discrete-time population models are usually formulated under the assumption that the dynamics of the total population size in generation $t$, denoted by $x(t)$, are governed by equations of the form

$$
x(t+1)=f(x(t))+\gamma x(t),
$$

where $\gamma \in(0,1)$ is the constant "probability" of surviving per generation, and $f: \mathbb{R}_{+} \rightarrow \mathbb{R}_{+}$models the birth or recruitment process [23].

Almost periodic effects can be introduced into Eq. (6.1) by writing the recruitment function or the survival probability as almost periodic sequences. This is modeled with the equation

$$
x(t+1)=f(t, x(t))+\gamma_{t} x(t),
$$

where either $\left\{\gamma_{t}\right\}_{t \in \mathbb{Z}_{+}}$or $f(t, x(t)) \in A P\left(\mathbb{Z}_{+}\right)$and each $\gamma_{t} \in(0,1)$.

In a recent paper, Franke and Yakubu, in [23], studied Eq. (6.2) with the periodic constant recruitment function

$$
f(t, x(t))=K_{t}\left(1-\gamma_{t}\right),
$$

and with the periodic Beverton-Holt recruitment function

$$
f(t, x(t))=\frac{\left(1-\gamma_{t}\right) \mu K_{t} x(t)}{\left(1-\gamma_{t}\right) K_{t}+\left(\mu-1+\gamma_{t}\right) x(t)},
$$

where the carrying capacity $K_{t}$ is $p$-periodic, $K_{t+p}=K_{t}$ for all $t \in \mathbb{Z}_{+}$and $\mu>1[10,23]$. Franke and Yakubu proved that, the periodically forced recruitment functions Eq. (6.3) and Eq. (6.4) generate globally attracting cycles in Eq. (6.2), see [23]. Here, we use Theorem 4.3 to show that when both $\left\{K_{t}\right\}_{t \in \mathbb{Z}_{+}}$and $\left\{\gamma_{t}\right\}_{t \in \mathbb{Z}_{+}}$ 
are almost periodic, then Eq. (6.2) supports a unique globally asymptotically stable almost periodic solution whenever the recruitment function is either Eq. (6.3) (almost periodic constant) or Eq. (6.4) (Almost periodic Beverton-Holt's model).

Theorem 6.1. Let

$$
f(t, x(t))=\frac{\left(1-\gamma_{t}\right) \mu K_{t} x(t)}{\left(1-\gamma_{t}\right) K_{t}+\left(\mu-1+\gamma_{t}\right) x(t)},
$$

where both $\left\{K_{t}\right\}_{t \in \mathbb{Z}_{+}}$and $\left\{\gamma_{t}\right\}_{t \in \mathbb{Z}_{+}}$are almost periodic, each $\gamma_{t} \in(0,1), K_{t}>0$ and $\mu>1$. Then Eq. (6.2) has a unique globally asymptotically stable almost periodic solution whenever

$$
\sup \left\{\left.\gamma_{t}\right|_{t \in \mathbb{Z}_{+}}\right\}<\frac{1}{\mu+1}
$$

Proof. Eq. (6.2) is in the form of Eq. (2.1), where

$$
A(t)=\gamma_{t}
$$

and

Consequently,

$$
f(t, x(t))=\frac{\left(1-\gamma_{t}\right) \mu K_{t} x(t)}{\left(1-\gamma_{t}\right) K_{t}+\left(\mu-1+\gamma_{t}\right) x(t)}
$$

$$
\begin{aligned}
|f(t, x)-f(t, y)| & \leq \frac{\left(1-\gamma_{t}\right)^{2} \mu K t^{2}|x-y|}{\left(1-\gamma_{t}\right)^{2} K_{t}^{2}+\left(\mu-1+\gamma_{t}\right)\left(1-\gamma_{t}\right) K_{t}(x+y)+\left(\mu-1+\gamma_{t}\right)^{2} x y} \\
& \leq \mu|x-y| .
\end{aligned}
$$

Hence, $f$ is Lipschitz with the Lipschitz constant $L=\mu$. Let $M=1$ and $\beta=\sup \left\{\left.\gamma_{t}\right|_{t \in \mathbb{Z}_{+}}\right\}$. Then, sup $\left\{\left.\gamma_{t}\right|_{t \in \mathbb{Z}_{+}}\right\}<\frac{1}{\mu+1}$ implies that

$$
\frac{M \beta L}{1-\beta}=\frac{\mu \cdot \sup \left\{\left.\gamma_{t}\right|_{t \in \mathbb{Z}_{+}}\right\}}{1-\sup \left\{\left.\gamma_{t}\right|_{t \in \mathbb{Z}_{+}}\right\}}<1
$$

and Eq. (4.3), is satisfied. Applying Theorem 4.6 yields the result.

Proceeding exactly as in the proof of Theorem 6.1 , it is easy to see that, when $f(t, x(t))=K_{t}\left(1-\gamma_{t}\right)$, then $f(t, x)-f(t, y)=0$ and the following result is immediate.

Corollary 6.2. Let $f(t, x(t))=K_{t}\left(1-\gamma_{t}\right)$, where both $\left\{K_{t}\right\}_{t \in \mathbb{Z}_{+}}$and $\left\{\gamma_{t}\right\}_{t \in \mathbb{Z}_{+}}$ are almost periodic, each $\gamma_{t} \in(0,1)$ and $K_{t}>0$. Then Eq. (6.2) has a unique globally asymptotically stable almost periodic solution whenever

$$
\sup \left\{\left.\gamma_{t}\right|_{t \in \mathbb{Z}_{+}}\right\}<1 \text {. }
$$

\section{Attenuance and Resonant Cycles in Almost Periodic ENVIRONMENTS}

Periodic environments usually diminish (respectively, enhance) populations via attenuant (respectively, resonant) stable cycles $[7,8,9,10],[13,14,15,16]$, and $[21,22,23,24]$. In this section, we focus on using precise mathematical definitions of attenuance and resonance to access evidence for attenuance or resonance in almost periodic environments. In particular, we prove that Model, Eq. (6.2), supports 
attenuant cycles when the recruitment function is the almost periodically forced Beverton-Holt model and the survival probability is constant (non-almost periodic).

To be precise, define

$$
M\left(a_{t}\right)=\lim _{i \rightarrow \infty}\left[\frac{a_{t+1}+\ldots+a_{t+i}}{i}\right]
$$

as the mean value of an almost periodic sequence $\left\{a_{t}\right\}$ on $\mathbb{Z}_{+}$. (see [25] for a similar proof for almost periodic sequence $\left\{a_{t}\right\}$ on $\mathbb{Z}$.) $M\left(a_{t}\right)$ is then called the mean value of $\left\{a_{t}\right\}$.

Lemma 7.1. Let $\left\{x_{t}\right\}$ be an almost periodic scalar sequence on $\mathbb{Z}_{+}$. Then $M\left(x_{t}\right)$ exists.

Proof. The proof is similar to the proof of Theorem 5, p. 690 in Kay Fan [18] and will be omitted.

Remark 7.2. The above proof of Lemma 7.1 can be extended to $\mathbb{R}^{n}$ or Banach spaces.

In Eq. (6.2), let

$$
f(t, x(t))=\frac{\left(1-\gamma_{t}\right) \mu K_{t} x(t)}{\left(1-\gamma_{t}\right) K_{t}+\left(\mu-1+\gamma_{t}\right) x(t)},
$$

where $\left\{K_{t}\right\}_{t \in \mathbb{Z}_{+}}$and $\left\{\gamma_{t}\right\}_{t \in \mathbb{Z}_{+}}$are almost periodic, and each $\gamma_{t} \in(0,1), K_{t}>0$ and $\mu>1$. By Corollary 6.2, Eq. (6.2) has a globally asymptotically almost periodic solution $\{\bar{x}(t)\}$ whenever $\sup \left\{\left.\gamma_{t}\right|_{t \in \mathbb{Z}_{+}}\right\}<\frac{1}{\mu+1}$.

Open Problem: Under what conditions do we have

$$
M(\bar{x}(t))<M\left(K_{t}\right) ? \quad \text { (attenuance) }
$$

Theorem 7.3. . In Eq. (6.2), let

$$
f(t, x(t))=\frac{\left(1-\gamma_{t}\right) \mu K_{t} x(t)}{\left(1-\gamma_{t}\right) K_{t}+\left(\mu-1+\gamma_{t}\right) x(t)},
$$

where $\left\{K_{t}\right\}_{t \in \mathbb{Z}_{+}}$is almost periodic, and each $\gamma_{t}=\gamma \in(0,1), K_{t}>0$ and $\mu>1$. Then,

(1) $M\left(K_{t}\right) \leq \sup \left\{\left.K_{t}\right|_{t \in \mathbb{Z}_{+}}\right\}<\infty$;

(2) $\lim _{t \rightarrow \infty} \sup \frac{1}{t} \sum_{t=0}^{t-1} x(t) \leq \lim _{t \rightarrow \infty} \sup \frac{1}{t} \sum_{t=0}^{t-1} K_{t}$;

for any solution $x(t)$. If $\bar{x}(t)$ is the unique almost periodic solution, then

$$
M(\bar{x}(t)) \leq M\left(K_{t}\right) .
$$

Proof. By Lemma 2.6, $\left\{K_{t}\right\}_{t \in \mathbb{Z}_{+}}$is almost periodic implies $\left\{K_{t}\right\}_{t \in \mathbb{Z}_{+}}$is bounded. Hence, $\sup \left\{\left.K_{t}\right|_{t \in \mathbb{Z}_{+}}\right\}<\infty$.

$$
M\left(K_{t}\right)=\lim _{i \rightarrow \infty} \frac{1}{i} \sum_{i=0}^{n-1} K_{i} \leq \lim _{i \rightarrow \infty} \frac{1}{i} i \sup \left\{\left.K_{t}\right|_{t \in \mathbb{Z}_{+}}\right\}=\sup \left\{\left.K_{t}\right|_{t \in \mathbb{Z}_{+}}\right\}<\infty .
$$


This establishes (1).

To prove (2), let $z(t)=\frac{1}{t} \sum_{i=0}^{t-1} x(i), K_{t}=\frac{1}{t} \sum_{i=0}^{t-1} K_{i}$.

$$
\begin{aligned}
z(t+1)= & \frac{1}{t+1} \sum_{i=0}^{t} x(i) \\
= & \frac{x(0)}{t+1}+\frac{1}{t+1} \sum_{i=0}^{t} x(i+1) \\
= & \frac{x(0)}{t+1}+\frac{1}{t+1} \sum_{i=0}^{t}\left[\frac{(1-\gamma) \mu K_{i} x(i)}{(1-\gamma) K_{i}+(\mu-1+\gamma) x(i)}+\gamma x(i)\right] . \\
z(t+1)= & \frac{x(0)}{t+1}+\frac{\gamma}{t+1} \sum_{i=0}^{t-1} x(i)+\frac{(1-\gamma) \mu}{(t+1)} \sum_{i=0}^{t-1} \frac{K_{i}}{(\mu-1+\gamma)}\left(\frac{(\mu-1+\gamma) x(i)}{(1-\gamma) K_{i}}\right) \\
= & \left.\frac{x(0)}{t+1}+\frac{\mu-1+\gamma}{t+1} z(t) K_{i}\right] \\
& +\sum_{i=0} K_{i} \frac{(i)}{(i+1)(\mu-1+\gamma)} \frac{\sum_{i=0}^{t-1} K_{i} h\left(\frac{(\mu-1+\gamma) x(i)}{(1-\gamma) K_{i}}\right)}{\sum_{i=0}^{t-1} K_{i}},
\end{aligned}
$$

where $h(t)=\frac{t}{1+t}, h^{\prime \prime}<0$.

Notice that $h$ is strictly convex and satisfies Jensen's inequality. Thus,

$$
\begin{aligned}
z(t+1)<\frac{x(0)}{t+1}+\frac{\gamma t}{t+1} z(t)+\frac{(1-\gamma) \mu}{(t+1)(\mu-1+\gamma)} \sum_{i=0}^{t-1} K_{i} h\left(\frac{\sum_{i=0}^{t-1} K_{i} \frac{(\mu-1+\gamma) x(i)}{(1-\gamma) K_{i}}}{\sum_{i=0}^{t-1} K_{i}}\right) \\
=\frac{x(0)}{t+1}+\frac{\gamma t}{t+1} z(t)+\frac{(1-\gamma) \mu t K_{t}}{(t+1)(\mu-1+\gamma)} \frac{\frac{1}{t} \sum_{i=0}^{t-1} \frac{K_{i}(\mu-1+\gamma)}{(1-\gamma) K_{i}} \frac{x(i)}{\sum_{r=0}^{n-1} K_{i}}}{\frac{1-1}{t} \sum_{i=0}^{t-1} K_{i}+\frac{1}{t} \sum_{i=0}^{t-1} \frac{\frac{K_{i}(\mu-1+\gamma)}{(1-\gamma) K_{i}} x(i)}{\sum_{i=0}^{t-1} K_{i}}} \\
z(t+1)<\frac{x(0)}{t+1}+\frac{\gamma t}{t+1} z(t)+\mu \frac{t}{t+1} K_{t} \frac{z(t)}{K_{t}+\frac{\mu-1+\gamma}{1-\gamma} z(t)} .
\end{aligned}
$$

Let $\lambda=\operatorname{limsupsup} z(t)$ and $\theta=\limsup K_{n}$. Then, there exists a sequence $t_{i} \rightarrow \infty$ such that $\lim _{t \rightarrow \infty} z\left(t_{i}+1\right)=\lambda$ and subsequence $\left\{z\left(t_{i}\right)\right\}$ and $\left\{K_{t_{i}}\right\}$ satisfying $\lim z\left(t_{i}\right) \leq \lambda$, and $\lim K_{t_{i}} \leq \theta$. 
Since $H(u, v)=\frac{u v}{u+v}$ is increasing in both arguments and $(0, \gamma)^{2}$, we have

$$
\begin{gathered}
\lambda=\lim _{i \rightarrow \infty} z\left(t_{i}+1\right) \leq \lim _{i \rightarrow \infty} \frac{x_{0}}{t_{i}+1}+\gamma \lambda+\frac{\mu \theta \lambda}{\theta+\frac{\mu-1+\gamma}{1-\gamma} \lambda} . \\
\text { Therefore, }(1-\gamma) \lambda \theta+(\mu-1+\gamma) \lambda^{2} \leq \mu \theta \lambda \\
\Rightarrow(\mu-1+\gamma) \lambda \leq(\mu-1+\gamma) \theta \\
\Rightarrow \lambda \leq \theta .
\end{gathered}
$$

Investigate attenuance and resonance of the general Beverton-Holt equation

$$
x_{n+1}=\frac{\left(1-\gamma_{n}\right) \mu K_{n} x_{n}}{\left(1-\gamma_{n}\right) K_{n}+\left(\mu-1+\gamma_{n}\right) x_{n}}+\gamma_{n} x_{n}
$$

$\gamma_{n}, K_{n} \in A P\left(\mathbb{Z}_{+}\right)$

\section{Population Models With Delay}

To study population models with constant delay in almost periodic environments, we consider delay difference equations. Our main concern in this section is to find sufficient conditions for the existence of a globally attracting almost periodic solution of the semilinear systems of difference equations with delay

$$
x(t+1)=A(t) x(t)+f(t, x(t-r), x(t-r+1), \ldots, x(t)), \quad t \in \mathbb{Z}_{+}
$$

where $A(t)$ is a continuous $k \times k$ almost periodic matrix defined on $\mathbb{Z}_{+}$and the function $f: \mathbb{Z}_{+} \times\left(\mathbb{R}^{k}\right)^{r+1} \mapsto \mathbb{R}^{k},(t, u) \mapsto f(t, u)$ is almost periodic in $t \in \mathbb{Z}_{+}$ uniformly in $u \in\left(\mathbb{R}^{k}\right)^{r+1}$. The proof of the following Lemma is straightforward.

Lemma 8.1. Let $x=\{x(t)\}_{t \in \mathbb{Z}_{+}}$be an $\mathbb{R}^{p}$-valued almost periodic sequence. Then the sequence defined by $y(t):=x(t-r)$ for all $t \geq r$, for some fixed $r \in \mathbb{Z}_{+}$, is almost periodic.

Proposition 8.2. Suppose that $f$ is globally Lipschitz with Lipschitz constant L. Then Eq. (8.1)has a unique globally asymptotically stable almost periodic solution whenever

$$
\frac{M \beta L \sqrt{r+1}}{1-\beta}<1
$$

where $M$ and $\beta$ are the dichotomy constants for the linear part of Eq. (8.1) and $r$ is the delay time.

\section{The Beverton-Holt's Model With Delay}

In this section, we study the Beverton-Holt model with delay in almost periodic environments. Thus, we consider the equation

$$
x(t+1)=\gamma_{t} x(t)+f(t, x(t-r), x(t-r+1), \ldots, x(t))
$$

for all $t \in \mathbb{Z}_{+}$, where $\left(\gamma_{t}\right)_{t \in \mathbb{Z}_{+}}$is an almost periodic sequence and $f: \mathbb{Z}_{+} \times$ $[0, \infty)^{r+1} \mapsto[0, \infty),(t, u) \mapsto f(t, u)$ is almost periodic in $t \in \mathbb{Z}_{+}$uniformly in $u=\left(u_{0}, u_{1}, \ldots, u_{r}\right) \in[0, \infty)^{r+1}$.

One requires the following assumption: 
(H) $f: \mathbb{Z}_{+} \times[0, \infty)^{r+1} \mapsto[0, \infty),(t, u) \mapsto f(t, u)$ is almost periodic in $t \in \mathbb{Z}_{+}$ uniformly in $u \in[0, \infty)^{r+1}$. Moreover, $f$ is Lipschitz in $u \in[0, \infty)^{r+1}$ uniformly in $t \in \mathbb{Z}_{+}$, i.e., there exists $L>0$ such that

$$
\left|f\left(t, u_{0}, u_{1}, \ldots, u_{r}\right)-f\left(t, v_{0}, v_{1}, \ldots, v_{r}\right)\right| \leq L \cdot\left(\sum_{k=0}^{r}\left|u_{k}-v_{k}\right|^{2}\right)^{1 / 2}
$$

for all $t \in \mathbb{Z}_{+}$and $\left(u_{0}, \ldots, u_{r}\right),\left(v_{0}, \ldots, v_{r}\right) \in[0, \infty)^{r+1}$.

For instance

$$
f(t, x)=\frac{\left(1-\gamma_{t}\right) \mu K_{t} x}{\left(1-\gamma_{t}\right) K_{t}+\left(\mu-1+\gamma_{t}\right) x}
$$

for $t \in \mathbb{Z}_{+}$and $x \in[0, \infty)$ satisfies assumption $(\mathbf{H})$ whenever both $\left(\gamma_{t}\right)_{t \in \mathbb{Z}_{+}}$and $\left(K_{t}\right)_{t \in \mathbb{Z}_{+}}$are almost periodic. In that case, $L=\mu$.

The constants of dichotomy related to Eq. (9.1) are respectively $M=1$ and $\beta=\sup _{t \in \mathbb{Z}_{+}} \gamma_{t} \in(0,1)$.

Corollary 9.1. Under assumption (H), Eq. (9.1) has a unique globally asymptotically stable almost periodic solution whenever

$$
\beta<\frac{1}{1+L \sqrt{r+1}}
$$

For instance if $L=1.01$, we then get the following approximations of $\beta_{0}$ in terms of $r$, the number of delays being considered:

\begin{tabular}{|c|c|}
\hline$r$ & $\beta_{0}=\frac{1}{1+1.01 \sqrt{r+1}}$ \\
\hline 0 & $\approx 0.4975$ \\
\hline 1 & $\approx 0.4118$ \\
\hline 2 & $\approx 0.3637$ \\
\hline 3 & $\approx 0.3311$ \\
\hline 4 & $\approx 0.3068$ \\
\hline 5 & $\approx 0.2878$ \\
\hline 6 & $\approx 0.2723$ \\
\hline$\ldots$ & $\ldots \ldots$. \\
\hline$\ldots$ & $\ldots \ldots$ \\
\hline 20 & $\approx 0.1776$ \\
\hline 100 & $\approx 0.0896$ \\
\hline
\end{tabular}

In view of the above, the following version of the Beverton-Holt equation with delay given by

$$
\begin{aligned}
x(t+1) & =\gamma_{t} x(t)+\frac{\left(1-\gamma_{t}\right) \mu K_{t} x(t-1)}{\left(1-\gamma_{t}\right) K_{t}+\left(\mu-1+\gamma_{t}\right) x(t)}, \quad t \geq 1 \\
x(0) & =x_{0}
\end{aligned}
$$

has a unique globally asymptotically stable almost periodic solution whenever

$$
\beta<\frac{1}{1+\mu \sqrt{2}}
$$


with $\mu>1$. However, if we consider the original version of Beverton-Holt equation with delay in the form

$$
\begin{aligned}
x(t+1) & =\gamma_{t} x(t)+\frac{\left(1-\gamma_{t}\right) \mu K_{t} x(t-1)}{\left(1-\gamma_{t}\right) K_{t}+\left(\mu-1+\gamma_{t}\right) x(t-1)}, \quad t \geq 1 \\
x(0) & =x_{0}
\end{aligned}
$$

we obtain a better result if we convert the equation into a two-dimensional system with no delay and apply Theorem 4.6 directly. It follows that this equation has a unique globally asymptotically stable almost periodic solution whenever

$$
\beta<\frac{1}{1+\mu}
$$

with $\mu>1$.

\section{CONCLUSiON}

Almost periodic deterministic models are more likely to capture the "noise" associated with real-population data. Using these models, we study the effects of almost periodic forcing of the carrying capacity, the demographic characteristic and survival rates of species on the long-term behavior of discretely reproducing populations. Others have studied the effects of periodic forcing of the carrying capacity, the demographic characteristic and survival rates of species on population dynamics [8, 9, 10], [12, 13, 14, 15, 16], [20, 21, 22, 23, 24], [28, 29, 30, 31], and $[34,35,36,37]$. Typically, such periodically forced models support attracting cycles that are either enhance via resonance or diminished via attenuance.

In almost periodic environments, simple population models support a globally attracting almost periodic solution. Developing a signature function for determining whether the almost periodic solution is attenuance or resonance is an interesting question that we plan to explore elsewhere.

\section{REFERENCES}

1. S. B. Angenent, The zero set of a parabolic differential equation, J. Reine Agnew. Math. 390 (1988), 79-96.

2. W. Arendt, C. Batty, M. Hieber, and F. Neubrander, Vector-valued Laplace Transforms and Cauchy Problems, Monographs in Mathematics, Birkhauser, 2001.

3. M. Begon, J. L. Harper and C. R. Townsend, Ecology: individuals, populations and communities, Blackwell Science Ltd (1996).

4. J. Blot and D. Pennequin, Existence and Structure Results on Almost Periodic Solutions of Difference Equations, J. Differ. Equations Appl.7 (2001), no. 3, 383-402

5. R. C. Casten and C. J. Holland, Instability results for reaction-diffusion equations with Neumann boundary conditions, J. Differential Equations. 27 (1978), no. 2, 266-273.

6. C. Corduneanu, Almost periodic functions, 2nd Edition, Chelsea-New York, 1989.

7. R. F. Costantino, J. M. Cushing, B. Dennis and R. A. Desharnais, Resonant popopulation cycles in temporarily fluctuating habitats, Bull. Math. Biol. 60(1998), 247-273.

8. J. M. Cushing, Periodic time-dependent predator-prey systems, SIAM J. Appl. Math. 32 (1977), 82-95.

9. J. M. Cushing, The LPA model, Fields Institute Communications 43 (2004), 29-55.

10. J. M. Cushing and S. M. Henson, Global dynamics of some periodically forced, monotone difference equations, J. Diff. Equations Appl. 7 (2001), 859-872.

11. S. N. Elaydi, Discrete Chaos. Chapman \& Hall/CRC, Boca Raton, FL (2000).

12. S. N. Elaydi, Periodicity and stability of linear Volterra difference equations, J. Math. Anal. Appl. 181(1994), 483-492. 
13. S. N. Elaydi and R. J. Sacker, Global Stability of Periodic Orbits of Nonautonomous Difference Equations and Population Biology, J. Differential Equations 208(2005), 258-273.

14. S. N. Elaydi and R. J. Sacker, Global Stability of Periodic Orbits of Nonautonomous Difference Equations in Population Biology and the Cushing-Henson conjectures, Proceedings of the Eighth International Conference on Difference Equations and Applications, Chapman \& Hall / CRC, Boca Raton, FL, 2005, 113-126.

15. S. N. Elaydi and R. J. Sacker, Global Stability of Periodic Orbits of Nonautonomous Difference Equations In Population Biology and Cushing-Henson Conjectures, J. Diff. Equations Appl. 11 (2005), no. 4-5, 337-346.

16. S. N. Elaydi and R. J. Sacker, Nonautonomous Beverton-Holt Equations and the CushingHenson Conjectures, J. Diff. Equations Appl. 11 (2005), 337-347.

17. S. N. Elaydi and A.-A. Yakubu, Global Stability of Cycles: Lotka-Volterra Competition Model With Stocking, J. Diff. Equations Appl. 8 (2002), no. 6, 537-549.

18. Ky Fan, Les fonctions asymptotiquement presque-périodiques d'une variable entière et leur application à l'étude de l'itération des transformations continues, Math. Z. 48 (1943), 685-711.

19. A. M. Fink, Almost Periodic Differential Equations, Lecture Notes in Mathematics 377, Springer-Verlag, New York-Berlin, 1974.

20. J. E. Franke and J. F. Selgrade, Attractor for Periodic Dynamical Systems, J. Math. Anal. Appl. 286 (2003), 64-79.

21. J. E. Franke and A.-A. Yakubu, Discrete-time metapopulation dynamics and unidirectional dispersal, J. Diff. Equations Appl. 9 (2003), no. 7, 633-653.

22. J. E. Franke and A.-A. Yakubu, Multiple Attractors Via Cusp Bifurcation In Periodically Varying Environments, J. Diff. Equations Appl. 11 (2002), no. 4-5, 365-377.

23. J. E. Franke and A.-A. Yakubu, Population models with periodic recruitment functions and survival rates, J. Diff. Equations Appl. 11 (2005), no. 14, 1169-1184.

24. J. E. Franke and A.-A. Yakubu, Signature functions for predicting resonant and attenuant population cycles. Bull. Math. Biol. (In press).

25. A. Halanay and V. Rasvan, Stability and stable oscillations in discrete time systems, Advances in Discrete Mathematics and Applications, Vol. 2, Gordon and Breach Science Publication, 2000.

26. M. P. Hassell, The dynamics of competition and predation, Studies in Biol. 72(1976), The Camelot Press Ltd.

27. D. Henry, Geometric theory of semilinear parabolic equations, Lecture Notes in Mathematics 840, Springer-Verlag, New York-Berlin, 1981.

28. S. M. Henson, Multiple attractors and resonance in periodically forced population models, Physics D. 140(2000), 33-49.

29. S. M. Henson, R. F. Costantino, J. M. Cushing, B. Dennis and R. A. Desharnais, Multiple attractors, saddles, and population dynamics in periodic habitats, Bull. Math. Biol. 61 (1999), 1121-1149.

30. S. M. Henson and J. M. Cushing, The effect of periodic habitat fluctuations on a nonlinear insect population model, J. Math. Biol. 36 (1997), 201-226.

31. S. M. Henson et al., Predicting dynamics of aggregate loafing behavior in Glaucose-Winged Gulls (Larus-Glaucoscens) at a Washington Colony, The AUK 121 (2004).

32. M. Hirsch, Attractors for discrete-time monotone systems in strongly ordered spaces, Geometry and topology, Lecture Notes in Mathematics, 1167, Springer, Berlin (1985).

33. R. Jajte, Almost periodic sequences. Colloq. Math. 12 1964/1965, 235-267.

34. D. Jillson, Insect populations respond to fluctuating environments, Nature 288(1980), 699700 .

35. V. L. Kocic, A note on nonautonomous Beverton-Holt model, J. Diff. Equations Appl., to appear.

36. R. Kon, A note on attenuant cycles of population models with periodic carrying capacity, $J$. Diff. Equations Appl., to appear.

37. R. Kon, Attenuant cycles of population models with periodic carrying capacity, J. Diff. Equations Appl., to appear.

38. J. Li, Periodic solutions of population models in a periodically fluctuating environment, Math. Biosc. 110 (1992), 17-25.

39. E. Liz and J. B. Ferreiro, A note on the global stability of generalized difference equations, Appl. Math. Lett 15 (2002), 655-659. 
40. R. M. May, Simple mathematical models with very complicated dynamics, Nature 261(1977), 459-469.

41. R. M. May, Stability and complexity in model ecosystems, Princeton University Press, 1974.

42. R. K. Miller, Almost periodic differential equations as dynamical systems with applications to the existence of a. p. solutions, Journal of Differential Equations,1 (1965).

43. A. Muchnik, A. Semonov, and M. Ushakov, Almost Periodic Sequences, Theoretical Comp. Sci. 304 (2003), 1-33.

44. D. Pennequin, Existence of Almost Periodic Solutions of Discrete Time Equations, Discrete and Continuous Dynamical Systems. 7 (2001), no. 1, 51-80.

45. W. E. Ricker, Stock and recruitment, Journal of Fisheries Research Board of Canada II(5) (1954), 559-623.

46. R. J. Sacker and G. Sell, Lifting properties in skew-product flows with applications to Differential Equations, Mem. Amer. Math. Soc. 11 (1977), no. 190, iv+67 pp.

Department of Mathematics, Howard University, 2441 6th Street N.W., Washington D.C. 20059

E-mail address: tdiagana@howard.edu

Department of Mathematics, Trinity University, San Antonio, TX 78212

E-mail address: selaydi@trinity.edu

Department of Mathematics, Howard University, 2441 6th Street N.W., Washington D.C. 20059

E-mail address: ayakubu@howard.edu 